\title{
Investigations of hippocampal astrocytes in lipopolysaccharide-preconditioned rats in the pilocarpine model of epilepsy
}

\author{
Jadwiga Jaworska-Adamu${ }^{1}$, Mirosława Dmowska ${ }^{2}$, Regina Cybulska ${ }^{1}$, \\ Aleksandra Krawczyk ${ }^{1}$, Bożena Pawlikowska-Pawlęga ${ }^{3}$ \\ ${ }^{1}$ Department of Animal Anatomy and Histology, Faculty of Veterinary Medicine, University of Life \\ Sciences, Lublin, Poland \\ ${ }^{2}$ Department of Cell Biology, Institute of Biology, M. Curie-Sklodowska University, Lublin, Poland \\ ${ }^{3}$ Department of Comparative Anatomy and Anthropology, Institute of Biology, \\ M. Curie-Sklodowska University, Lublin, Poland
}

\begin{abstract}
The present paper is the first work to determine the effect of lipopolysaccharide (LPS) in the pilocarpine model of epilepsy on the morphology of rat hippocampal astrocytes in vivo. The study involved adult male Wistar rats, which 72 hours prior to administration of pilocarpine hydrochloride (PILO) were intraperitoneally (ip) preconditioned with LPS at a dose of $0.5 \mathrm{mg} / \mathrm{kg}$ b.w. The control animals were administered (ip) saline or LPS alone. The astrocytes in the control animals displayed characteristic stellate morphology. Examinations of the astrocytes were performed on days one, three and 21 of the pilocarpine model of epilepsy (i.e. in the acute, silent and chronic periods). The astrocytes of the CA1 and CA3 pyramidal layers of the hippocampus were observed and analyzed at the structural and ultrastructural levels. It was demonstrated that on days one and three, glial cells from both the nonpreconditioned and the LPS-preconditioned animals displayed similar reactive changes, manifesting themselves as swelling of cell bodies, glial processes, and astrocytosis. Moreover, reduction in cell organelles aggregated at one pole and the presence of vacuoles were observed. The most pronounced astrogliosis and cell swelling occurred on day 21. We conclude that LPS has no effect on the morphology of astrocytes in the pilocarpine model of epilepsy, unlike the results obtained by other authors in vitro. (Folia Histochemica et Cytobiologica 2011; Vol. 49, No. 2, pp. 219-224)
\end{abstract}

Key words: epilepsy, astrocytes, lipopolysaccharide, hippocampus, rat

\section{Introduction}

Unlike neurons, astrocytes are more resistant to various sources of damage to the central nervous system (CNS). Recently, there has been growing interest in the glial cells, as they may play a key role in the epileptogenic process. Long-term investigations have

\footnotetext{
Correspondence address: J. Jaworska-Adamu, Department of Animal Anatomy and Histology, Faculty of Veterinary Medicine, University of Life Sciences, Akademicka Str. 12, 20-950 Lublin, Poland; tel.: (+ 48 81) 44567 41;

e-mail: jadwiga.jaworska@up.lublin.pl
}

failed to determine whether the enhanced activation of astrocytes is a favorable phenomenon, or whether it enhances neuronal changes in various pathological conditions $[1,2]$.

The cells in question play numerous neuroprotective roles: they maintain ion homeostasis and appropriate concentration of neurotransmitters in the extracellular space. They also participate in synaptic transmission, fulfil trophic functions, and regulate the blood/brain barrier function [3]. During epileptic seizures, sharply increasing glutamate concentration in the extracellular space has a neurotoxic effect, and ion (especially calcium) homeostasis and energy metabolism are disturbed [4]. Excitotoxic neuronal death 
occurs in acute pathological conditions, for instance in epileptic seizure attacks, brain ischemic stroke, mechanical injury or hypoglycemia. During glutamateinduced neuronal death, astrocytes are capable of taking up the neurotransmitter to a certain degree, which indicates their neuroprotective function. It has been demonstrated in mixed neuron and astrocyte cultures that the uptake of glutamate by the glial cells may not be sufficient to prevent neuronal damage. Activated astrocytes may release various growth factors which maintain neuronal survival $[5,6]$. And when substantial amounts of glutamate are accumulated in the extracellular space, the activated astrocytes enhance neurotoxicity by cytokine and nitrogen oxide production. Pathological factors induce immediate morphological and functional changes in the cells, which have been referred to as 'reactive astrocytes'. This is manifested as hypertrophy i.e. swelling of glial cell bodies and processes aimed at compensation of the changes in the extracellular environment. Shortterm swelling may be a transient and reversible phenomenon, whereas long-term swelling may lead to astrocyte death. When detrimental factors operate over a longer period, astrocytes may acquire new traits related to the synthesis of numerous proteins. This phenomenon is referred to as hyperplasia. Long-term morphological changes in astrocytes may induce proliferation i.e. astrogliosis, and migration of these cells into damaged neurons [7].

The morphology of astrocytes has been characterized and compared in the acute and chronic period of various models of epilepsy. Considerable astrogliosis has been reported on days 1-3 and in weeks 2-3 after administration of pilocarpine and kainic acid (KA). Two to three weeks after KA injection into the hippocampus, increased immunoreactivity of the glial fibrillary acidic protein (GFAP) was observed. Normal astrocyte morphology was shown after 90 days in KA-induced epilepsy [8].

We ourselves have found that short-term LPS preconditioning may increase neuronal tolerance to subsequent damage (unpublished data). However, the function of hippocampal astrocytes after preconditioning with the bacterial LPS endotoxin in the pilocarpine epilepsy model in animals has not been investigated to date. Thus, the aim of our study was to examine the morphology of astrocytes of the CA1 and CA3 pyramidal layers of the hippocampus after LPS-preconditioning, and to compare it to the morphology in non-preconditioned and control animals.

\section{Material and methods}

The experiments were performed on 144 adult male Wistar rats (180-240 g) purchased from the Animal Breeding Laboratory
(Warsaw, Poland). The animals were kept under controlled light and temperature conditions ( 12 hours light/12 hours dark cycle; $20-21^{\circ} \mathrm{C}$ ), with free access to water and food for two weeks prior to the experiment and during the experiment.

The experiment was carried out according to the Polish government regulations concerning experiments on animals (Dz.U. 97.111.724) and the European Community Council Directive of 24 November 1986 (86/609/EEC). All efforts were made to minimize animal suffering and the number of animals required. The protocols were approved by the First Local Ethical Committee in Lublin (374/2002).

The control groups consisted of 48 animals. One control group was intraperitoneally (ip) given a solvent (saline), while the second group (preconditioned rats) was administered LPS (Sigma) at a dose of $0.5 \mathrm{mg} / \mathrm{kg}$ b.w. In two experimental groups (96 animals), seizures were induced by intraperitoneal administration of single dose pilocarpine hydrochloride (PILO, Sigma, $380 \mathrm{mg} / \mathrm{kg}$ b.w.). In order to avoid discomfort caused by peripheral muscarinic receptor activation, all the animals had previously been treated with scopolamine methylbromide (Sigma, $1 \mathrm{mg} / \mathrm{kg}$ b.w. sc, $30 \mathrm{~min}-$ utes before PILO injection).

Half of the experimental rats were pharmacologically preconditioned with LPS at a dose of $0.5 \mathrm{mg} / \mathrm{kg}$ b.w., 72 hours before PILO injection. Mortality after PILO seizures was about $20 \%$. The animals which survived were anesthetized with ip $50 \mathrm{mg} / \mathrm{kg}$ b.w. Nembutal (Abbott, England) after days 1, 3 and 21. On the basis of changes in the animals' behavior, there are three characteristic periods of status epilepticus (SE). The acute period persists to 24 hours, the silent period from 24 hours to five days, and the chronic period from five to 51 days [9-11].

Light microscopic investigations. The animals were perfused via the left ventricle with freshly prepared $4 \%$ paraformaldehyde. The hippocampus was removed and routinely immersed in paraffin, and then dissected $(4,230$ $-3,750 \mu \mathrm{m}$, according to the atlas of König and Kippel [12]) on the microtome into $10 \mu \mathrm{m}$ slices, which were subsequently stained with cresyl violet. All the samples were observed and photographed under the Axiolab light microscope (Zeiss).

Electron microscopic investigations. The rats were perfused via the left heart ventricle with a mixture containing $0.5 \%$ paraformaldehyde and $1.5 \%$ glutaraldehyde in $0.1 \mathrm{M}$ phosphate buffer $\mathrm{pH}$ 7.4. The brains were then removed, immersed in $0.8 \%$ paraformaldehyde plus $2.5 \%$ glutaraldehyde in $0.1 \mathrm{M}$ phosphate buffer for two hours and dissected into thin sections. After rinsing in cacodylate buffer $\mathrm{pH} 7.4$, the specimens were post-fixed in $1 \%$ osmium tetroxide for another two hours, then dehydrated in graded alcohol-acetone solutions and embedded in Spurr. Ultrathin sections were cut with a glass knife on microtome RMC MT-XL (Tucson, AZ, USA), collected on cooper grids and contrasted 
with the use of $9 \%$ uranyl acetate for 20 minutes followed by Reynold's liquid (lead nitrate and sodium citrate) for 10 minutes. The samples were observed under LEO-Zeiss 912 AB electron microscope (Oberkochen, Germany).

\section{Results}

\section{Observations of astrocytes under the light microscope}

Normal astrocyte morphology was observed in the pyramidal layer of the hippocampal fields CA1 and $\mathrm{CA} 3$ in the control animals, and those preconditioned with LPS alone. Glial cells were located in the vicinity of the cell bodies of the pyramidal neurons; they displayed stellate shapes and had nuclei in the central part of the light cytoplasm (Figures 1A, 2A).

After days 1 and 3 of the pilocarpine epilepsy model, reactive (mainly hypertrophic) astrocytes were observed in the pyramidal layer of the hippocampal fields CA1 and CA3 in the non-preconditioned and LPS-preconditioned rats. The majority of the analyzed astrocytes displayed swelling of the light cell bodies adjacent to the pyramidal neurons which preserved a normal structure. In the large nuclei of some glial cells, the presence of chromosomes indicated mitotic divisions (Figure 1B). Moreover, on day 3, single shrunk neurons with dark cytoplasm surrounded by light, swollen astrocyte processes were present in the pyramidal layer of fields CA1 and CA3 in the non-preconditioned animals.

On day 21 of the pilocarpine epilepsy model, the biggest changes in the CA1 and CA3 field astrocytes were observed in the non-preconditioned and in LPS-preconditioned animals, compared to the other rat groups. These glial cells displayed hypertrophy, which was manifested in swelling of the bodies, glial processes and, occasionally, of the cell nuclei. In most of the study cells, the light cytoplasm contained two, or even three, spherical cell nuclei, which implied intense gliosis. Hypertrophic reactive astrocytes adhered to normal and morphologically changed neurons. In LPS-preconditioned animals, fewer changed neurons were observed (data not reported) (Figures 1C, 2B, 2C).

\section{Observations of astrocytes under the electron microscope}

In the control and LPS alone preconditioned animals in the pilocarpine epilepsy model, the astrocytes in the pyramidal CA1 and CA3 layers had a normal ultrastructure. The small amount of electron-light cytoplasm contained the rough endoplasmic reticulum, mitochondria. In the central part of the cells there was an oval or round cell nucleus with chromatin accumulated at the envelope (Figure 3A).

In the non-preconditioned and LPS-preconditioned rats, most hypertrophic astrocytes with swollen bodies were observed in the pyramidal layer of fields CA1 and CA 3 on days 1 and 3 of the pilocarpine epilepsy model. The cytoplasm of the swollen astrocytes displayed considerable reduction in the cell organelles. The heterochromatin in the oval and round nuclei was clustered beneath the nuclear envelope. On days 1 and 3, in both the non-preconditioned and the LPS-preconditioned animals, preparation for divisions was visible in some astrocytes, where chromosomes were seen in the nuclei. Additionally, numerous cells contained vacuoles of various volumes (Figures 3B, 3C).

On day 21, similar changes were still observed in the astrocytes of the non-preconditioned and LPS-preconditioned rats in the pilocarpine epilepsy model. The cell bodies were still swollen, and the light cytoplasm contained numerous vacuoles. The mitochondria were characterized by electron-dense matrix, and the endoplasmic reticulum cisterns were reduced. Astrocytosis involving mutual adherence of the young astrocytes was frequently observed in the microscopic images (Figure 3D).

\section{Discussion}

Our study revealed that administration of LPS alone did not induce changes in the morphology of astrocytes of the CA1 and CA3 pyramidal layers of the hippocampus. Recent in vitro studies have demonstrated that, 72 hours after LPS had been added to the incubation medium, astrocytes in the mouse forebrain lost their flat shape and became typical, activated stellate cells with elongated processes and acquired new traits, i.e. they displayed expression of the glial fibrillary acidic protein (GFAP). Furthermore, LPS contributed to enhanced activity and expression of the glial membrane excitatory amino acid transporter GLT-1 (EAAT2) [13], which indicates that increased expression of the astrocytic GLT-transporter protects neurons against the toxicity of excess glutamate in the extracellular space. LPS alone administered to animals may activate astrocytes to produce and release growth factors, e.g. the nerve growth factor (NGF), the brain-derived neurotrophic factor (BDNF), the glial-derived neurotrophic factor (GDNF), the fibroblastic growth factor (FGF2) and other proteins. It has been suggested that glial cells have a neuroprotective function in the case of inconsiderable neuronal damage [14].

Only a few studies performed in vitro on cultured rat astrocytes have demonstrated LPS-triggered stim- 
A

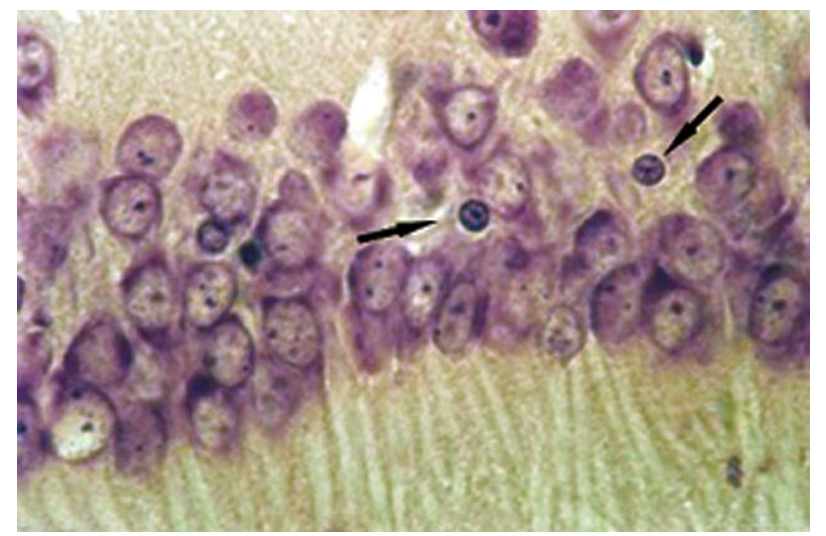

B

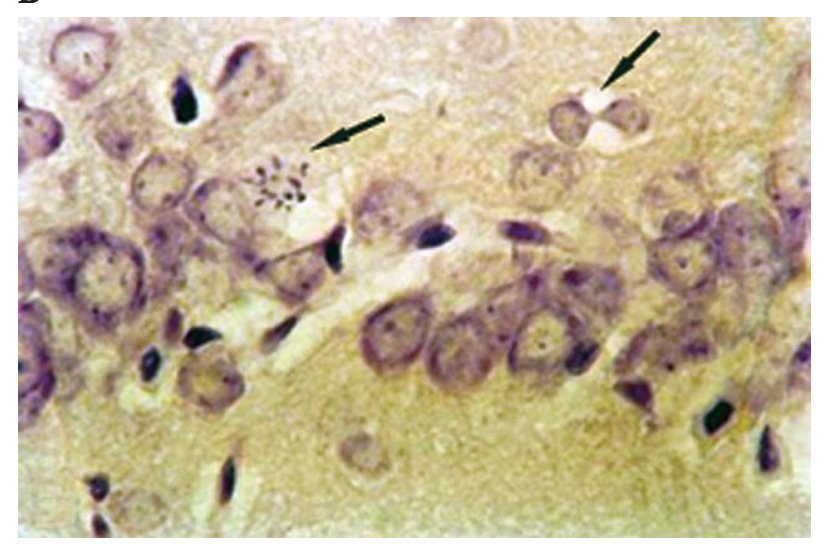

\section{C}

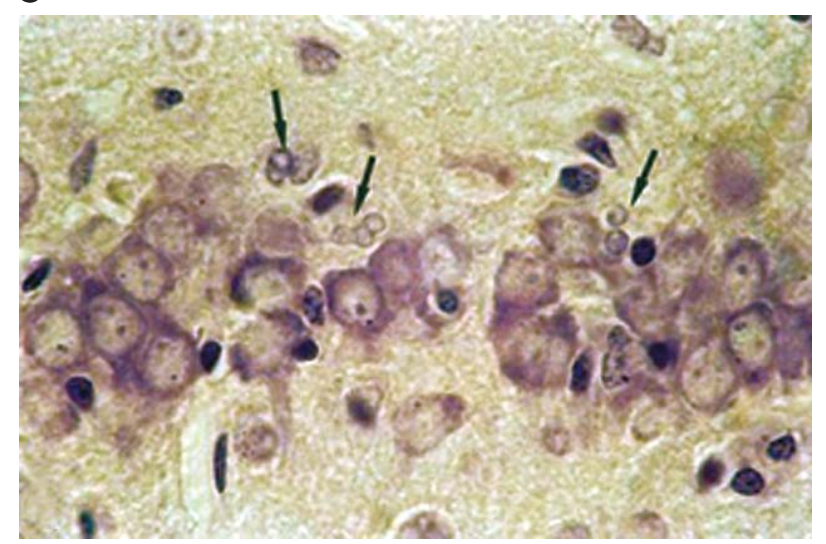

Figure 1. Astrocytes $(\downarrow)$ of the pyramidal layer of hippocampal field CA1(A) in a control rat. (B) LPS-preconditioned rat after day 3 of the pilocarpine model of epilepsy. (C) Non-preconditioned rat after day 21 of the pilocarpine model of epilepsy (original magnification $\times 1,000)$

ulation of these cells to produce the tumor necrotic factor (TNF) and of interleukin 1 (IL 1) and interleukin 6 (IL 6) genes. Increased pro-inflammatory cytokine production displayed by these cells may play a crucial role in the pathogenesis of some diseases of the central nervous system induced by interaction
A

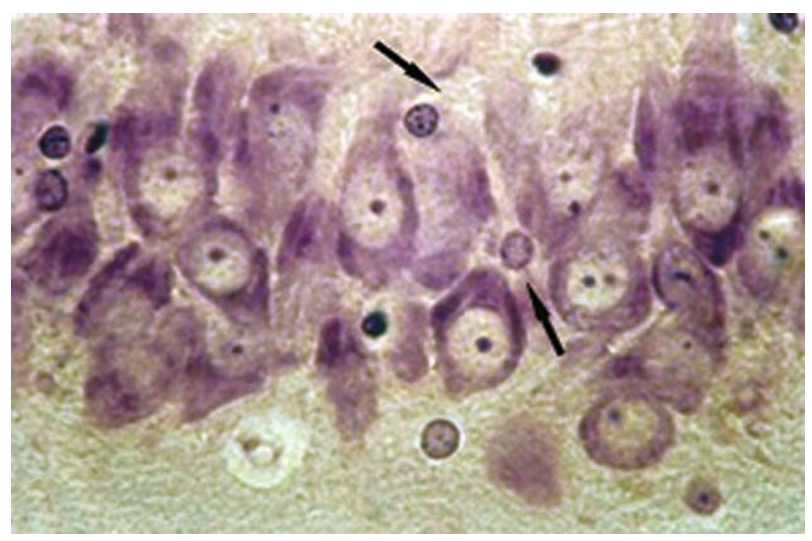

B

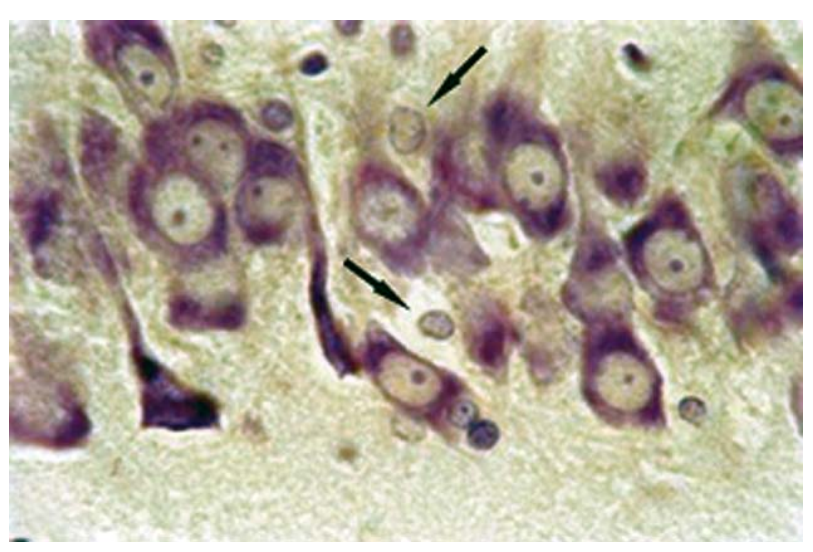

C

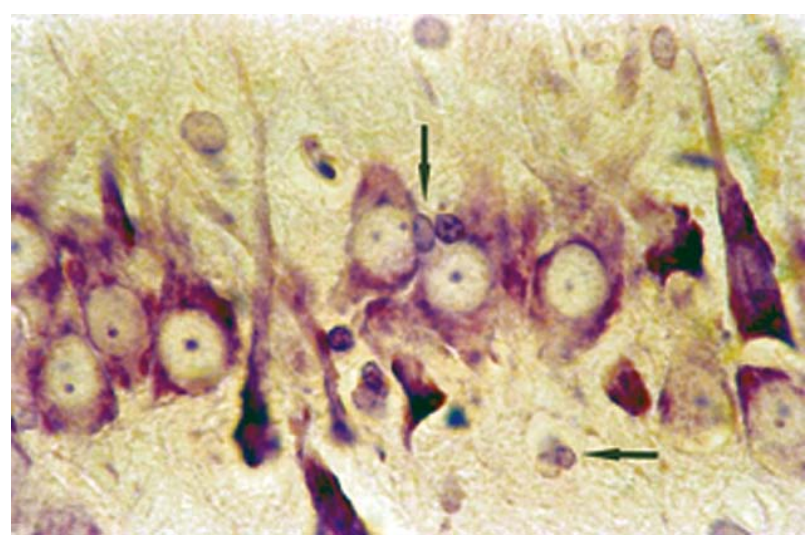

Figure 2. Astrocytes $(\downarrow)$ of the pyramidal layer of hippocampal field CA3 (A) in a control LPS-preconditioned rat. (B) Non-preconditioned rat after day 21 of the pilocarpine model of epilepsy. (C) LPS-preconditioned rat after day 21 of the pilocarpine model of epilepsy (original magnification $\times 1,000$ )

between the nervous and the immune systems. It is assumed that astrocytes may enhance neuronal degenerative changes through release of pro-inflammatory cytokines $[15,16]$. During epilepsy, astrocytes may be activated before neuron degeneration takes place. It has not been determined whether neuron 
A

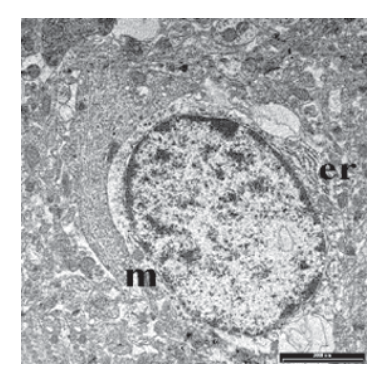

C

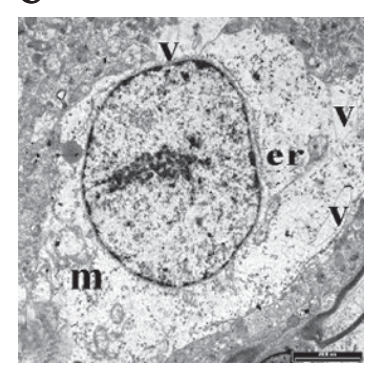

B

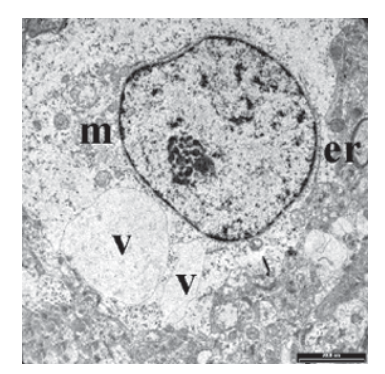

D

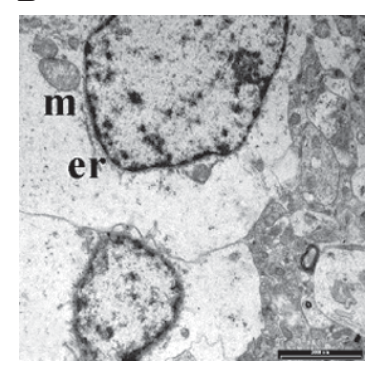

Figure 3. Electronograms of astrocytes from the pyramidal layer of hippocampal fields. Astrocytes from field CA1 (A) cell with normal morphology, ergastoplasm (er) and mitochondria $(\mathrm{m})$ in a control rat, $(\mathbf{B})$ non-preconditioned rat after day 3 of the pilocarpine model of epilepsy.

A swollen cell body with electron-light cytoplasm and aggregation of organelles at one pole at the cell nucleus. Few mitochondria (m), partially preserved ergastoplasm (er) and large vacuoles (v), (C) LPS-preconditioned rat after day 3 of the pilocarpine model of epilepsy. A swollen cell body with electron-light cytoplasm, swollen mitochondria (m), scanty ergastoplasm (er) accumulated in the vicinity of the nucleus, and vacuoles (v). A small amount of chromatin in the nucleus accumulated near the envelope, a visible metaphase plate. (D) Fragments of two adherent astrocytes from hippocampal field CA3 in an LPS-preconditioned rat after day 21 of the pilocarpine model of epilepsy The light, swollen cytoplasm contains few cell organelles: mitochondria (m) and few ergastoplasmic cisterns (er) at the nucleus (scale bar 2,000 nm)

degeneration is a primary factor in astrocyte activation or vice versa. In the reactive astrocytes of the entorhinal cortex, hippocampal field CA1 and amygdaloid nuclei, GFAP and vimentin immunoreactivity in immature astrocytes increases before degenerative changes in the neurons occur. This supports intense glia proliferation. Also, the changes in astrocytes are believed to be related to inhibited neuronal activity [17].

Our own study revealed similarities in the astrocyte morphology in the pyramidal layer of hippocampal fields CA1 and CA3 on days 1 and 3 in the nonpreconditioned and LPS-preconditioned animals in the pilocarpine model of epilepsy. In both cases, swelling of the glial bodies and processes, loss of cell organelles and astrocytosis were observed.

This suggests that reactive astrocytes respond with almost immediate swelling, i.e. with hypertrophy and proliferation. Astrogliosis may affect regulation of homeostasis in the extracellular space. Markedly increased aquaporin 4 expression in the membrane water channels was shown in these glial cells after ischemia. The swelling of the astrocytes observed very early after both pilocarpine epilepsy and preconditioning may improve the encephalon water metabolism. Numerous factors, such as a high glutamate level, high concentration of potassium ions, lactic acid and free oxygen radicals, contribute to astrocytic swelling [18-20].

In status epilepticus (SE), i.e. approximately on days $1-3$, immediate activation of astrocytes and microglia [8] and similar astrogliosis were demonstrated after KA administration to the rat hippocampus $[21,22]$. Two to three weeks after KA application, the astrocytes displayed changed morphology, but there were no changes after 90 days. In contrast, young astrocytes were present in the pilocarpine-induced experimental epilepsy model after 60 days [8].

In our own study, on day 21 we observed numerous mitotic divisions and swollen bodies of young astrocytes in the preconditioned and non-preconditioned animals in the pyramidal layer of hippocampal fields CA1 and CA3.

We conclude that LPS, as demonstrated in our own studies, does not substantially affect the morphology of astrocytes. This contradicts the results obtained by other authors in vitro on cultured rat astrocytes with LPS added to the incubation medium.

\section{Acknowledgements}

This study was supported by a grant from the Rector of UMCS for Scientific Research and International Co-operation, Lublin, Poland.

\section{References}

1. Chen Y, Swanson RA. Astrocytes and brain injury. J Cerebr Blood Flow Metab. 2003;23:137-149.

2. Keyser JD, Mostert JP, Koch MW. Dysfunctional astrocytes as key players in the pathogenesis of central nervous system disorders. J Neurol Sci. 2008;267:3-16.

3. Schwacz R. Early glial dysfunction in epilepsy. Epilepsia (Suppl. 2). 2008;49:1-2.

4. Szydłowska K, Kamińska B. Pharmacological modulation of function and viability of astrocytes as a novel neuroprotective strategy in stroke. Kosmos. 2008;57:315-329.

5. Brown DR. Neuronal release of vasoactive intestinal peptide is important to astrocytic protection of neurons from glutamate toxicity. Mol Cell Neurosci. 2000;15:465-475.

6. Rossi DJ, Brady JD, Mohr C. Astrocyte metabolism and signaling during brain ischemia. Nat Neurosci. 2008;10:1377-1386.

7. Janeczko K. The proliferative activity of astrocytes after immunoglobulin uptake in the injured mouse cerebral hemisphere. Folia Histochem Cytobiol. 1994;32:239-241. 
8. Garzillo CL, Mello EAM. Characterization of reactive astrocytes in the chronic phase of the pilocarpine epilepsy. Epilepsia (Suppl). 2002;43:107-109.

9. Turski WA, Cavalheiro EA, Bortolotto ZA, Mello LM, Schwarz M, Turski L. Seizures produced by pilocarpine in mice: a behavioural, electroencephalographic and and morphological analysis. Brain Res. 1984;321:237-253.

10. Turski WA, Cavalheiro EA, Schwarz M, Czuczwar SJ, Kleinrok Z, Turski L. Limbic seizures produced by pilocarpine in rats: behavioural, electroencephalographic and neuropathological study. Behav Brain Res. 1983;9:315-335.

11. Klitgaard H, Matagne A, Vneste-Goemaere J, Margineanu DG. Pilocarpine-induced epileptogenesis in the rat: impact of initial duration of status epilepticus on electrophysiological and neuropathological alterations. Epilepsy Res. 2002;51:93-107.

12. König FR, Klippel RA. The rat brain. A stereotaxic atlas of the forebrain and lower parts of the brain stem. RR Krieger, Huntington NY 1970.

13. O'Shea RD, Lau ChL, Farso MC et al. Effects of lipopolysaccharide on glial phenotype and activity of glutamate transporters: evidence for delayed up-regulation and redistribution of GLT-1. Neurochem Int. 2006;48:604-610.

14. Takuma K, Baba A, Matsuda T. Astrocytes apoptosis: implications for neuroprotection. Progress Neurobiol. 2004; 72:111-127.
15. Lieberman AP, Pitha PM, Shin HS, Shin ML. Production of tumor necrotic factor and other cytokines by astrocytes stimulated with lipopolysaccharide or a neurotropic virus. Proc Natl Acad Sci. 1999;86:6348-6352.

16. Hu S, Martella A, Anderson WR, Chao ChC. Role of cytokines in lipopolysaccharide-induced functional and structural abnormalities of astrocytes. Glia. 2004;10:227-234.

17. Khurgel M, Switzer RC $3^{\text {rd }}$, Teskey GC, Spiller AE, Go I. Activation of astrocytes during epileptogenesis in the absence of neuronal degeneration. Neurobiol Dis. 1995;2: 23-35.

18. Rogowski MA. Astrocytes get in the act in the epilepsy. Nat Med. 2005;11:919-920.

19. Pera J. Hartowanie przez niedokrwienie. Aktualności neurologiczne (sympozjum udar mózgu). 2006;2:17-25.

20. Dirnagl U, Piller J. Focal cerebral ischemia: the multifaceted role of glial cells. Neuroglia, Oxford University Press, 2005.

21. Babb TL, Mathern GW, Pretorius JK et al. Astrocytes may contribute to the latent period in progressive neurons loss, axon sprouting, and chronic seizure in rat kainate hippocampal epilepsy. Epilepsy Res Suppl. 1996;12:343-354.

22. Shapiro LA, Wang L, Ribak ChE. Rapid astrocyte and microglial activation following pilocarpine-induced seizures in rats. Epilepsia. 2008;49:33-41.

Submitted: 16 February, 2010 Accepted after reviews: 30 January, 2011 